\title{
24-Hour Urinary Calcium in Primary Hyperparathyroidism
}

\author{
Carrie E. Black, MD; Richard L. Berg, MS; and Andrew C. Urquhart, MD
}

\begin{abstract}
Objective: To establish whether urinary and serum calcium levels are correlated in patients with primary hyperparathyroidism (PHPT) and to evaluate related factors including serum levels of parathyroid hormone (PTH) and vitamin D, age, gender and renal function.

Design: Retrospective review on patients undergoing a parathyroidectomy for PHPT from 20002008. Data collected included: age, sex, preoperative serum calcium (ionized and total), creatinine, PTH, postoperative ionized calcium, vitamin D, and 24-hour urine calcium and creatinine. Data were evaluated by standard descriptive statistics.

Results: Of the 214 patients evaluated, preoperative 24-hour urinary calcium was available for 150 patients (70\%). 24-hour urine calcium levels did not correlate significantly with preoperative total serum or ionized calcium, PTH, vitamin D, or postoperative serum ionized calcium. There were significant correlations $(P<0.000 \mathrm{I})$ of 24 -hour urinary calcium with age, serum creatinine, and urine creatinine. Secondary analysis grouped subjects by urinary calcium level greater or less than $400 \mathrm{mg} / 24$ hours. Age and urinary creatinine were significantly different between the two groups.

Conclusions: In patients with PHPT who underwent parathyroidectomy, there was little correlation between preoperative 24-hour urine calcium and preoperative serum calcium, PTH, or vitamin D levels. 24-hour urine calcium levels were correlated with renal function and age, but these correlations are likely to occur in the general population and are not specific to this disease group.
\end{abstract}

Keywords: Hypercalciuria; Primary hyperparathyroidism; 24-hour urine calcium

$\mathrm{T}$ he normal physiology of calcium metabolism is complex and depends on many factors such as renal function, diet, and age. When this normal metabolism is disrupted, it can acutely lead to hypercalcemia, which causes an increase in urinary calcium excretion through mediation of the calcium sensing receptor (CaSR) in the kidney. Hypercalciuria has been used as a diagnostic marker and indicator for parathyroidectomy in patients with primary hyperparathyroidism (PHPT). Urinary calcium excretion as a function of serum calcium was demonstrated more than 50

Corresponding Author: Andrew Urquhart, MD; Otolaryngology - Head and Neck Surgery; Marshfield Clinic; 1000 North Oak Avenue; Marshfield, WI 54449; Phone: (7I5) 387-5245; Fax: (7I5) 389-5757; Email: urquhart. andrew@marshfieldclinic.org years ago. ${ }^{1}$ However, it is not clear if 24-hour urine calcium levels correlate with preoperative total serum or ionized calcium, parathyroid hormone (PTH), and vitamin D in patients with primary hyperparathyroidism.

The 24-hour urinary sample, in addition to serologic studies, has historically been part of the standard diagnostic evaluation for patients suspected of PHPT. The use of 24-hour urine studies in PHPT has served two primary functions: elevated urinary calcium was thought to be a risk factor for
Received: March 22, 2013

Revised: June 5, 2013

Accepted: July 17, 2013

doi: $10.3121 / \mathrm{cmr} .2013 .1164$
Grant Support: Supported by

Marshfield Clinic Research Foundation

Disease Specific Restricted Funds. 
Table 1. Descriptive summary of the laboratory values.

\begin{tabular}{lccc}
\hline & N & Mean & SD \\
\hline Serum Calcium (Total), mg/dL & 149 & 10.7 & 0.66 \\
Serum Calcium (lonized), mg/dL & 133 & 6.0 & 0.49 \\
Serum BUN, mg/dL & 135 & 17.7 & 7.8 \\
Serum Creatinine, mg/dL & 146 & 1.02 & 0.87 \\
Serum PTH, pg/mL & 149 & 138.1 & 130.8 \\
Urine Calcium, mg/24hr & 150 & 327.0 & 182.2 \\
Urine Creatinine, mg/24hr & 150 & 1.25 & 0.48 \\
Total Serum Vitamin D, ng/mL & 101 & 33.3 & 12.2 \\
Postoperative Calcium (lonized), mg/dL & 150 & 5.1 & 0.40 \\
\hline
\end{tabular}

BUN, blood urea nitrogen; PTH, parathyroid hormone

nephrolithiasis, and 24-hour urine calcium studies have been used to distinguish asymptomatic PHPT from familial hypocalciuric hypercalcemia (FHH). However, guidelines published in the Journal of Clinical Endocrinology and Metabolism in 2009 no longer recommend hypercalciuria as a criterion for parathyroid surgery. ${ }^{2}$ The omission of urinary calcium in the updated guidelines for PHPT stems from new perspectives on the pathogenesis of nephrolithiasis. In the absence of nephrolithiasis, hypercalciuria alone is not considered to be an indication for surgery for PHPT; however, other surgeons still regard urinary calcium excretion of $>400 \mathrm{mg}$ an indication for surgery. ${ }^{2,3}$

The other role for urinary calcium studies in the workup of hypercalcemia is to rule out $\mathrm{FHH}$, which is caused by a mutation that inactivates the CaSR, a G protein-coupled receptor expressed in the parathyroid gland and renal tubule. Patients with FHH typically present with hypercalcemia, normal to mildly elevated PTH, and hypocalciuria. ${ }^{4-6}$ If there is a strong suspicion for $\mathrm{FHH}$, the presence of the mutation in the CaSR gene can be detected using readily available polymerase chain reaction techniques. FHH is an asymptomatic, benign disease; thus, it is important to make the distinction from PHPT, since parathyroidectomy is not indicated in this condition. ${ }^{7,8}$

For most patients presenting in our practice for evaluation of parathyroidectomy in the setting of primary hyperparathyroidism, a 24-hour urinary calcium level is obtained during the preoperative workup. Given the changing guidelines, with urinary calcium levels no longer a criterion for parathyroidectomy, we questioned the need for 24-hour calcium levels in patients with PHPT and wondered whether the urine calcium levels could be predicted from the elevated serum calcium levels. Therefore, we sought to determine if there was a correlation between 24-hour urinary calcium levels and serum and urinary laboratory markers relevant in this disease process.

\section{Methods}

The study was approved by Institutional Review Board of Marshfield Clinic prior to data collection. Marshfield Clinic is a large multi-specialty, multi-site group practice with regional centers located throughout central and northern Wisconsin. Surgeries for primary hyperparathyroidism performed in the years 2000 through 2008 by one surgeon (ACU) were reviewed. Data were extracted from the Marshfield Clinic electronic medical record and included age, sex, preoperative serum calcium (ionized and total), creatinine, $\mathrm{PTH}$, vitamin D, blood urea nitrogen (BUN), 24-hour urine creatinine, 24-hour urine calcium, and postoperative ionized serum calcium.

Prior to analyses, the electronic data were reviewed, and missing and extreme values were manually reviewed for quality assurance purposes. Standard descriptive statistics were used to summarize the demographic characteristics of the study subjects and their laboratory results. Graphical

Table 2. Correlations with 24-hour urinary calcium.

\begin{tabular}{lccc}
\hline & N & Spearman correlation & $\boldsymbol{P}$ value \\
\hline Age & 150 & -0.393 & $<0.0001$ \\
Serum Calcium (Total), $\mathrm{mg} / \mathrm{dL}$ & 149 & 0.130 & 0.114 \\
Serum Calcium (lonized), mg/dL & 133 & 0.168 & 0.053 \\
Serum Creatinine, $\mathrm{mg} / \mathrm{dL}$ & 146 & -0.357 & $<0.0001$ \\
Serum PTH, pg/mL & 149 & -0.004 & 0.960 \\
Urine Creatinine, g/24hr & 150 & 0.457 & $<0.0001$ \\
Total Serum Vitamin D, ng/mL & 101 & -0.202 & 0.043 \\
\hline
\end{tabular}

PTH, parathyroid hormone 


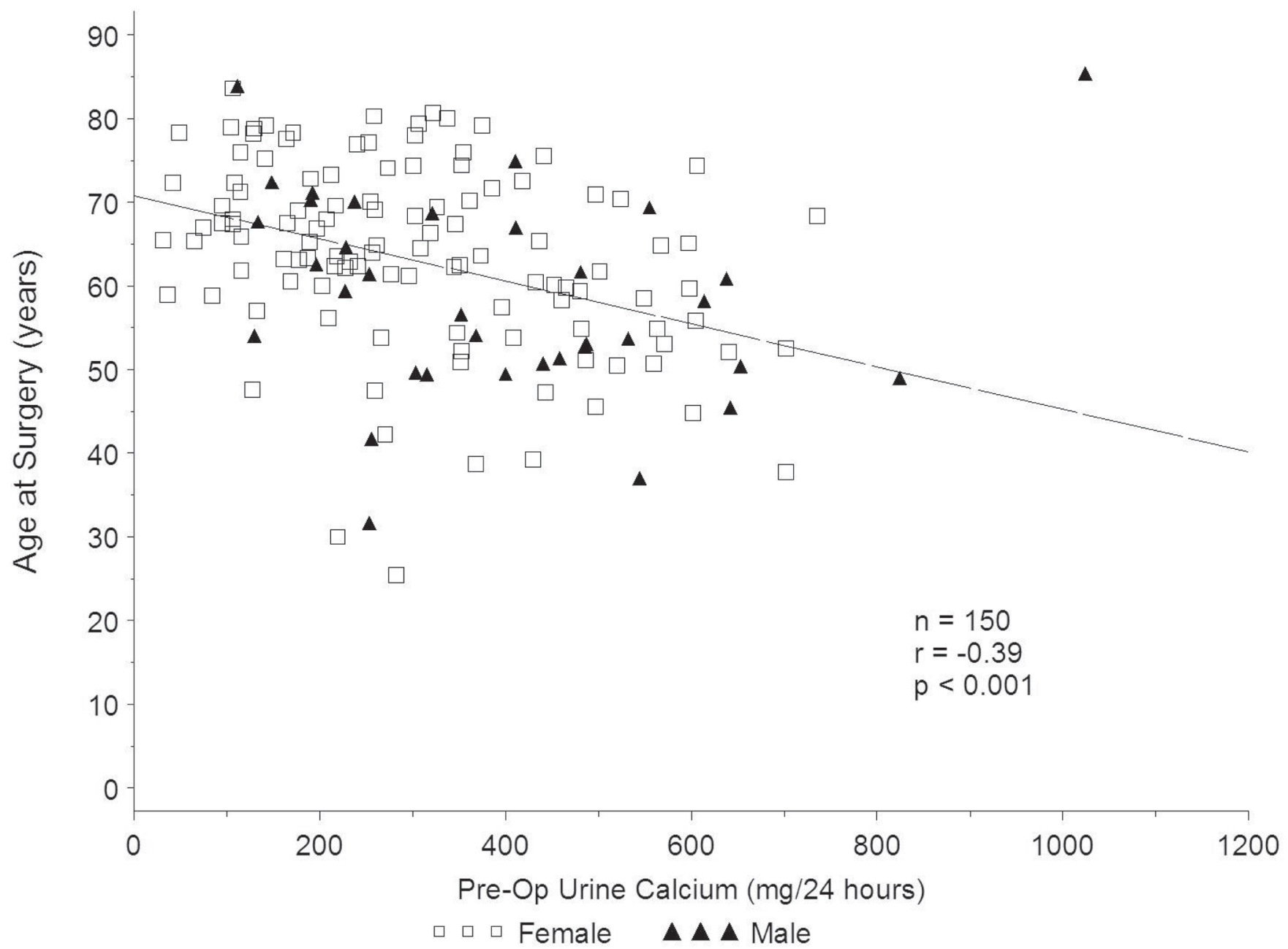

Figure 1. Age at surgery versus preoperative urine calcium. Older patients had lower urine calcium compared to the younger population.

review showed skewed distributions for laboratory results (particularly serum creatinine, PTH, and BUN), and the primary analyses were based on nonparametric (rank-based) procedures. The Spearman rank correlation was used to evaluate associations among the measures, and the Wilcoxon signed-rank test was used to compare results by level of urinary calcium (less than $400 \mathrm{mg} / 24$ hours or not). Analyses were conducted using SAS version 9.2 statistical software. Results were deemed statistically significant at the 5\% level $(P<0.05)$ without adjustments for multiple comparisons.

\section{Results}

A total of 214 patients were evaluated, and 24-hour urinary calcium levels were available in 150 patients. Patients with and without urine calcium were found to be similar with respect to demographics and other laboratory results, and the analyses presented here will be limited to those with urine calcium. These patients included 115 females $(77 \%)$ and 35 males $(23 \%)$, with ages ranging from 25 to 85 years (median 63 years). The mean preoperative total calcium level was 10.7 $\mathrm{mg} / \mathrm{dL}$, and the mean preoperative ionized calcium level was $6.0 \mathrm{mg} / \mathrm{dL}$. The mean postoperative ionized calcium level was $5.1 \mathrm{mg} / \mathrm{dL}$. The mean preoperative 24-hour urinary calcium level was $327 \mathrm{mg} / 24$ hours, with the upper quartile being $452 \mathrm{mg} / 24$ hours (table 1 ).

Relatively low correlations were observed for 24-hour urinary calcium levels with preoperative total calcium $(P=0.11)$, ionized calcium $(P=0.05)$, PTH $(P=0.96)$, and vitamin D $(P=0.04)$ (table 2$)$. These correlations were expected, based on known trends with hypercalcemia and related effects. There were highly significant correlations $(P<0.0001)$ of 24-hour urinary calcium with age (figure 1), urine creatinine (figure 2), and serum creatinine levels (figure 3). The correlations were similar in females and males analyzed separately (data not shown).

In a secondary analysis, subjects were divided into two groups based on whether or not urinary calcium was less than $400 \mathrm{mg} / 24$ hours (table 3 ). In the group with urinary calcium $\geq 400 \mathrm{mg} / 24$ hours, the mean age was about 8 years younger than the other group, with a higher percentage male $(34.7 \%$ vs. $17.8 \%$ ). Additionally, the urinary creatinine was found to be significantly different between the two groups: the group with urinary calcium $\geq 400 \mathrm{mg} / 24$ hours had a mean urine creatinine of $1.5 \mathrm{~g} / 24$ hours, as compared to the other group 


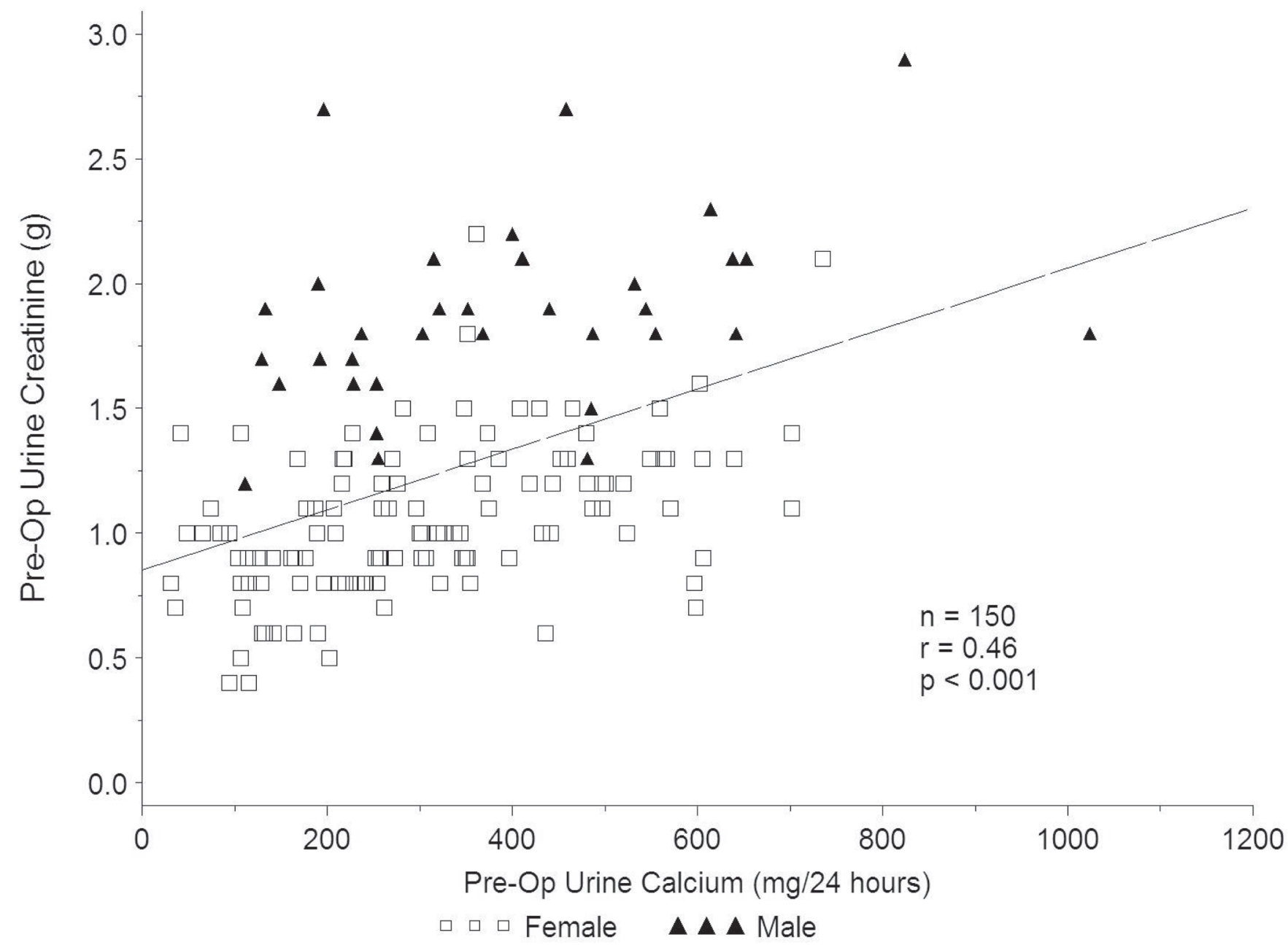

Figure 2. Preoperative urine creatinine versus preoperative urine calcium. Characterizes the significant positive correlation between preoperative urine calcium and preoperative urine creatinine.

with a mean urine creatinine of $1.1 \mathrm{~g} / 24$ hours. There was no significant difference between the two groups when evaluated for serum calcium, creatinine, BUN, PTH, vitamin D, or postoperative calcium levels. In models with covariates to adjust for age and gender differences, urine creatinine remained as the only laboratory result showing a statistically significant group difference (data not shown).

\section{Discussion}

Patients with normal renal function typically excrete approximately $100 \mathrm{mg}$ to $300 \mathrm{mg}$ of urinary calcium per day. Hypercalciuria, defined by the National Institutes of Health guidelines in 1990 and 2002 as $400 \mathrm{mg}$ calcium/24 hours in urine collected at home on a free diet, has been used as both a diagnostic marker and an indication for parathyroidectomy in patients with PHPT $^{8-10}$ This recommendation stemmed from the assumption that hypercalciuria posed a significant risk for the development of nephrolithiasis. However, a study by Berger and colleagues ${ }^{11}$ demonstrated that there was no significant correlation between the 24-hour urinary calcium and the serum calcium, in comparing a cohort of PHPT patients who presented with nephrolithiasis to those who did not. Patients with PHPT and stones usually have moderate to severe hypercalciuria but only mild to moderate increase in serum calcium. Pre- and postoperative 24-hour urine collection between non-stone formers did not show any differences with any variables. ${ }^{11,12}$ In addition, there are mixed results from studies that have tried to demonstrate that hypercalciuria itself promotes nephrolithiasis. ${ }^{13}$ Given this information, the guidelines for the management of asymptomatic PHPT have recently changed. A 24-hour urine calcium is still recommended in the initial evaluation of suspected patients with PHPT, in order to differentiate it from $\mathrm{FHH}$, but is no longer recommended as a criterion for parathyroidectomy.

The urinary studies (24-hour total urine calcium, calcium/ creatinine clearance ratio, and fasting urinary calcium excretion [spot urine]) were also historically used to make the distinction between FHH and PHPT. A value of $<100 \mathrm{mg}$ /day for total urinary calcium was considered diagnostic for $\mathrm{FHH}$; however, this value was set arbitrarily, and many patients undergoing parathyroidectomy with a confirmed adenoma on exploration have urine calcium excretion values similar to or below this cutoff. Also, the 24-hour urinary calcium study has the potential for inaccuracy due to age, renal function, varied 


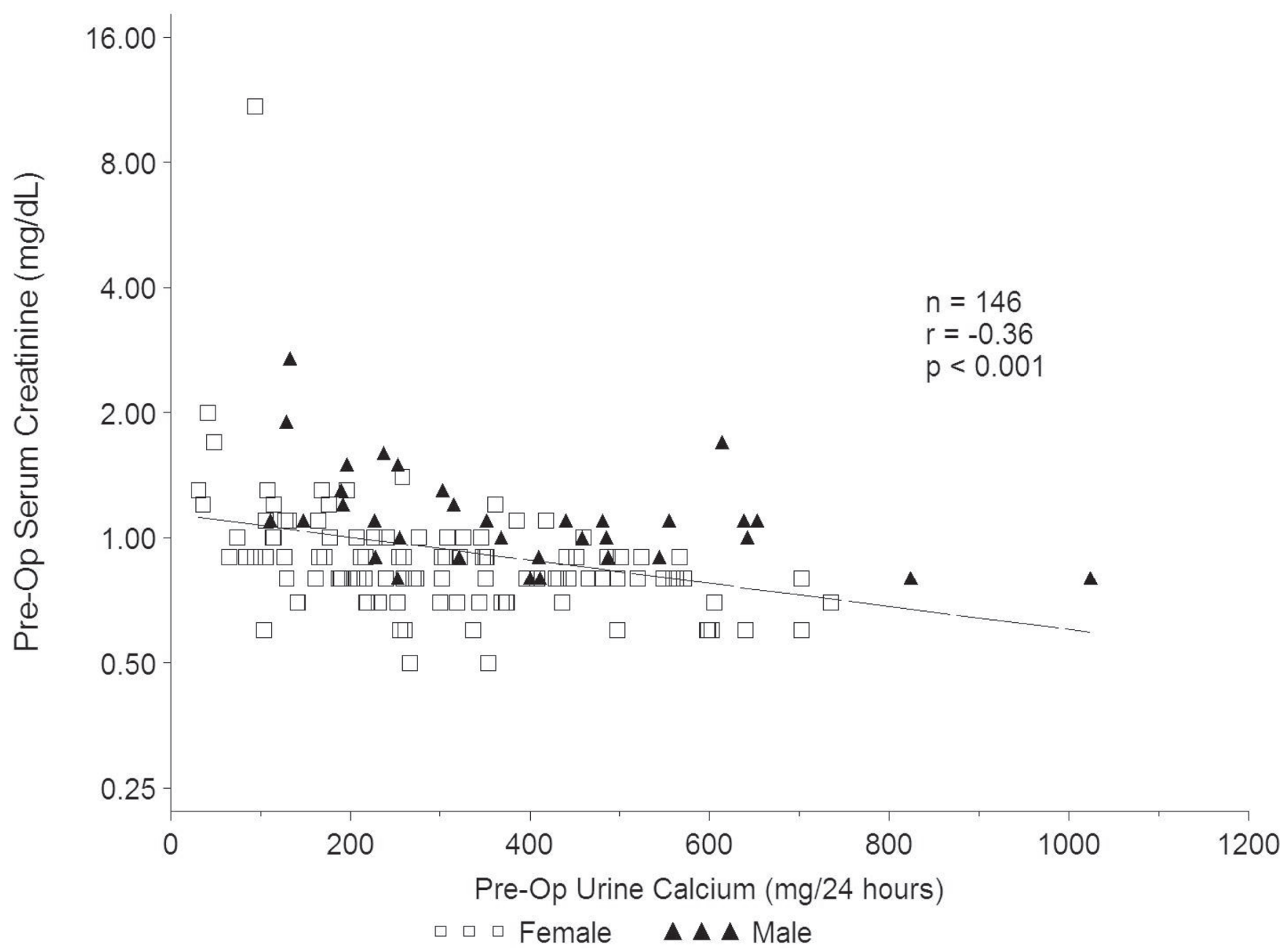

Figure 3. Preoperative serum creatinine versus preoperative urine calcium. Shows the significant negative correlation between preoperative serum creatinine and preoperative urine calcium.

dietary calcium intake, medications that affect the amount of calcium excreted, and the reliance on patient compliance. ${ }^{3}$ Because of the difficulty with urine studies in general, some have advocated that rather than 24-hour urinary studies, estimated glomerular filtration rate (eGFR) should be used to determine the level of kidney function in PHPT. An eGFR of less than $60 \mathrm{~mL} / \mathrm{min}$ should be used as a marker, because at this level of function PTH begins to rise in patients with chronic kidney disease. ${ }^{14}$ Also, the National Kidney Foundation no longer recommends the use of 24-hour urinary collections for estimation of renal function, but rather recommends the Modification of Diet in Renal Disease (MDRD) study calculation \#7, which is a modification of the Cockcroft-Gault equation that includes race, albumin, and urea nitrogen for a more accurate estimate of GFR. ${ }^{14}$ Similarly, the calcium/creatinine $(\mathrm{Ca} / \mathrm{Cr})$ clearance ratio, also calculated from 24-hour urine collections, has been found to be relatively non-specific. ${ }^{15}$ A ratio less than 0.01 was considered diagnostic for $\mathrm{FHH}$, while a ratio greater than 0.02 was thought to be typical for PHPT. 6,16 However, in follow-up studies, approximately one-third of patients with confirmed PHPT had a clearance ratio in the range of $0.01-0.02 .^{4,5,14,17,18}$ The $\mathrm{Ca} / \mathrm{Cr}$ clearance ratio is also used as a marker of hypercalciuria, where hypercalciuria cannot be excluded if the urinary creatinine concentration exceeds $40 \mathrm{mg} / 100 \mathrm{~mL} \cdot{ }^{19,20} \mathrm{In}$ the evaluation of PHPT, it is recommended that FHH be ruled out, even though this condition is very rare. Currently, if there is a strong suspicion for $\mathrm{FHH}$, the presence of the CaSR gene mutation can be detected using readily available polymerase chain reaction techniques. This genetic sequencing provides diagnostic security when the plot of spot urinary calcium excretion to PTH is indicative of FHH. However, this should be reserved for cases in which treatment would be significantly altered based on the diagnosis..$^{5,7,14,15,19}$ Hypercalcemia increases eGFR; however, tubular resorption of calcium is altered by many other factors such as age, medications, and hormones. While the volume of urine collected can affect the urinary creatinine and calcium levels and could be affected by diuretic use, we did not specifically analyze these factors. It could be a contributing factor to explain the correlation between older patients and lower urine calcium levels, particularly if there was a higher use of diuretics in this group. Our mean preoperative 24-hour urinary calcium levels were relatively higher than mean serum calcium levels of $10.2 \mathrm{mg} /$ $\mathrm{dL}$, but, there was little correlation between preoperative 24-hour urine calcium and preoperative serum calcium, PTH, 
Table 3. Comparisons of two groups based on level of urine calcium.

\begin{tabular}{|c|c|c|c|c|c|c|c|}
\hline & \multicolumn{3}{|c|}{ Urine Calcium $<400 \mathrm{mg} / 24 \mathrm{hr}$} & \multicolumn{3}{|c|}{ Urine Calcium $\geq 400 \mathrm{mg} / 24 \mathrm{hr}$} & \multirow[b]{2}{*}{$P$ value } \\
\hline & $\mathbf{N}$ & Mean & SD & $\mathbf{N}$ & Mean & SD & \\
\hline Age & 101 & 65.1 & 11.4 & 49 & 57.6 & 10.3 & $<0.001$ \\
\hline Serum Calcium (Total), mg/dL & 101 & 10.7 & 0.68 & 48 & 10.7 & 0.63 & 0.919 \\
\hline Serum Calcium (lonized), mg/dL & 89 & 5.9 & 0.48 & 44 & 6.1 & 0.50 & 0.105 \\
\hline Serum Creatinine, mg/dL & 99 & 1.1 & 1.04 & 47 & 0.9 & 0.19 & 0.008 \\
\hline Serum PTH, pg/mL & 100 & 141.1 & 150.4 & 49 & 131.9 & 77.6 & 0.819 \\
\hline Urine Creatinine, $g / 24 \mathrm{hr}$ & 101 & 1.1 & 0.42 & 49 & 1.5 & 0.50 & $<0.001$ \\
\hline Total Serum Vitamin D, ng/mL & 70 & 34.4 & 13.5 & 31 & 30.9 & 8.6 & 0.124 \\
\hline
\end{tabular}

or vitamin D levels. Urinary calcium levels were, however, strongly correlated with age, and also serum and urine creatinine, as demonstrated in figures 1 through 3. Figure 1 shows that older patients had lower urine calcium compared to the younger population; figure 2 characterizes the significant positive correlation between urine calcium and urine creatinine; and figure 3 shows that there is a negative correlation between serum creatinine and urine calcium. The 24-hour urine calcium correlates with serum creatinine and urine creatinine because complexed (calcium bound to phosphate, citrate, carbonate, and other anions) and ionized calcium is freely filterable in the glomerulus as urinary ultrafiltrate, and $98 \%$ is reabsorbed along the renal tubule. Increased urinary creatinine (reflected by increased creatinine clearance and GFR) likely explain the increase in calcium excretion. This has been previously demonstrated in pregnant women. ${ }^{21}$ Also, renal insufficiency, reflected by decreased creatinine clearance, decreases renal calcium filtration. We know that age is negatively correlated with GFR, which results in decreased urinary calcium levels, as noted in our study. All of these relationships would be expected in the general population, and are therefore not specific to patients with PHPT. These findings also support the new guidelines for the evaluation of PHPT, where hypercalciuria is no longer regarded as an indication for surgery. ${ }^{2}$

\section{Conclusion}

In patients with PHPT, urinary calcium levels are related to renal function and age, but show no correlation with serum calcium, PTH, or vitamin D levels. These relationships are demonstrated in the general population and are not specific to this disease process.

\section{Acknowledgements}

The authors thank Drs. Narayana Murali, Ram Pathak, and Michael Caldwell of the Marshfield Clinic for expert advice. They also thank the Marshfield Clinic Research Foundation's Office of Scientific Writing and Publication for assistance in the preparation of this manuscript.

\section{References}

1. Nordin BE, Peacock M. Role of kidney in regulation of plasmacalcium. Lancet 1969:2:12801283.
2. Bilezikian JP, Khan AA, Potts JT Jr; Third International Workshop on the Management of Asymptomatic Primary Hyperthyroidism. Guidelines for the management of asymptomatic primary hyperparathyroidism: summary statement from the third international workshop. J Clin Endocrinol Metab 2009;94:335-339.

3. Silverberg SJ, Lewiecki EM, Mosekilde L, Peacock M, Rubin MR. Presentation of asymptomatic primary hyperparathyroidism: proceedings of the third international workshop. J Clin Endocrinol Metab 2009;94:351-365.

4. Fraser WD. Hyperparathyroidism. Lancet 2009;374:145-458.

5. Guarnieri V, Canaff L, Yun FH, Scillitani A, Battista C, Muscarella LA, Wong BY, Notarangelo A, D'Agruma L, Sacco M, Cole DE, Hendy GN. Calcium-sensing receptor (CASR) mutations in hypercalcemic states: studies from a single endocrine clinic over three years. J Clin Endocrinol Metab 2010;95:1819-1829.

6. Riccardi D, Brown EM. Physiology and pathophysiology of the calcium-sensing receptor in the kidney. Am J Physiol Renal Physiol 2010;298:F485-F499.

7. Pidasheva S, Canaff L, Simonds WF, Marx SJ, Hendy GN. Impaired cotranslational processing of the calcium-sensing receptor due to signal peptide missense mutations in familial hypocalciuric hypercalcemia. Hum Mol Genet 2005;14:1679-1690.

8. AACE/AAES Task Force on Primary Hyperparathyroidism. The American Association of Clinical Endocrinologists and the American Association of Endocrine Surgeons position statement on the diagnosis and management of primary hyperparathyroidism. Endocr Pract 2005;11:49-54.

9. Bilezikian JP, Potts JT Jr, Fuleihan Gel-H, Kleerekoper M, Neer R, Peacock M, Rastad J, Silverberg SJ, Udelsman R, Wells SA. Summary statement from a workshop on asymptomatic primary hyperparathyroidism: a perspective for the $21 \mathrm{st}$ century. J Clin Endocrinol Metab 2002;87:5353-5361.

10. NIH conference. Diagnosis and management of asymptomatic primary hyperparathyroidism: consensus development conference statement. Ann Intern Med 1991;114:593-597.

11. Berger AD, Wu W, Eisner BH, Cooperberg MR, Duh QY, Stoller ML. Patients with primary hyperparathyroidism--why do some form stones? J Urol 2009;181:2141-245.

12. Sorensen MD, Duh QY, Grogan RH, Tran TC, Stoller ML. Differences in metabolic urinary abnormalities in stone forming and nonstone forming patients with primary hyperparathyroidism. Surgery 2012;151:477-483.

13. Rejnmark L, Vestergaard P, Mosekilde L. Nephrolithiasis and renal calcifications in primary hyperparathyroidism. J Clin Endocrinol Metab 2011;96:2377-2385. 
14. Eastell R, Arnold A, Brandi ML, Brown EM, D'Amour P, Hanley DA, Rao DS, Rubin MR, Goltzman D, Silverberg SJ, Marx SJ, Peacock M, Mosekilde L, Bouillon R, Lewiecki EM. Diagnosis of primary hyperparathyroidism: proceedings of the third international workshop. J Clin Endocrinol Metab 2009;94:340-350.

15. Christensen SE, Nissen PH, Vestergaard P, Heickenforff L, Brixen K, Mosekilde L. Discriminative power of three indices of renal calcium excretion for the distinction between familial hypocalciuric hypercalcaemia and primary hyperparathyroidism: a follow-up study on methods. Clin Endocrinol (Oxf) 2008;69:713-720.

16. Bilezikian JP, Silverberg SJ. Clinical practice. Asymptomatic primary hyperparathyroidism. N Engl J Med 2004;350:17461751.

17. Marx SJ, Attie MF, Levine MA, Spiegel AM, Downs RW Jr, Lasker RD. The hypocalciuric or benign variant of familial hypercalcemia: clinical and biochemical features in fifteen kindreds. Medicine (Baltimore) 1981;60:397-412.

18. Law WM Jr, Heath H 3rd. Familial benign hypercalcemia (hypocalciuric hypercalcemia). Clinical and pathogenetic studies in 21 families. Ann Intern Med 1985;102:511-519.

19. Gunn IR, Gaffney D. Clinical and laboratory features of calcium-sensing receptor disorders: a systematic review. Ann Clin Biochem 2004;41:441-458.

20. Gunn IR, Wallace JR. Urine calcium and serum ionized calcium, total calcium and parathyroid hormone concentrations in the diagnosis of primary hyperparathyroidism and familial benign hypercalcaemia. Ann Clin Biochem 1992;29:52-58.

21. Howart AT, Morgan DB, Payne RB. Urinary excretion of calcium in late pregnancy and its relation to creatinine clearance. Am J Obstet Gynecol 1977;129:499-502.

\section{Author Affiliations}

Carrie E. Black, MD ${ }^{*}$; ; Richard L. Berg, $M S^{*}$; and Andrew C. Urquhart, $M D^{*}$

*Department of General Surgery, Marshfield Clinic, Marshfield Clinic, Marshfield, WI

Biomedical Informatics Research Center, Marshfield Clinic Research Foundation, Marshfield, WI

Department of Otolaryngology- Head and Neck Surgery, Marshfield Clinic, Marshfield, WI

${ }^{a}$ Current affiliation: Department of Plastics and Reconstructive Surgery, University of Minnesota, Minneapolis, MN 